\title{
SOBRE O CONHECIMENTO PROFISSIONAL DOCENTE NO DESENVOLVIMENTO DA APRENDIZAGEM DE ALUNOS DOS ANOS INICIAIS EM MATEMÁTICA
}

\section{KNOWLEDGE ABOUT TEACHING PROFESSIONAL DEVELOPMENT IN THE EARLY YEARS OF LEARNING IN MATHEMATICS STUDENTS}

\author{
Edvonete Souza de Alencar * \\ Pontifícia Universidade Católica - São Paulo (*) \\ E-mail: edvonete.s.alencar@hotmail.com
}

\begin{abstract}
Resumo
$\mathrm{O}$ artigo apresenta uma pesquisa que teve como cenário investigativo uma escola que obteve avanço de proficiência nos anos de 2008 a 2012 em uma avaliação externa. Nosso objetivo foi investigar quais são os possíveis conhecimentos profissionais docentes são necessários para que ocorra a aprendizagem dos alunos dos anos iniciais do Ensino Fundamental. Aplicamos um questionário, uma entrevista e recolhemos atividades de um professor do $5^{\circ}$ ano, segmento avaliado. Referenciamo-nos aos autores do Conhecimento profissional Docente: Shulman, Ball e Bass e Tardif, assim como a Vergnaud para nos embasar quanto ao objeto matemático. Percebemos que o estudo e uso adequado dos materiais disponibilizados pela Secretária da Educação, assim como a complementação com atividades contextualizadas são fatores significativos no desempenho dos alunos desta escola.
\end{abstract}

Palavras-chave: Educação Matemática, Conhecimento Profissional Docente e Aprendizagem

\begin{abstract}
The article presents an investigative scenario that had as a school that got advance proficiency in the years 2008-2012 in an external evaluation. Our goal was to investigate what are the possible professional knowledge teachers are needed for student learning in the early years of elementary school occurs. We applied a questionnaire, interview and collect a professor at $5^{\circ}$ year, reported segment activities. Referenciamo us the authors of Professional Teaching Knowledge: Shulman, Ball and Bass and Tardif, like Vergnaud to support us as the mathematical object. We realize that the study and proper use of materials provided by the Secretary of Education, as well as complementation with contextualized activities are significant factors in the performance of the students of this school.
\end{abstract}

Keywords: Mathematics Education, Teacher Professional Knowledge and Learning. 


\section{INTRODUÇÃO}

O presente artigo foi elaborado tendo em vista analisar e buscar mais aspectos relevantes que nos levam a inferir quais são os possíveis conhecimentos necessários para que ocorra a aprendizagem dos alunos dos anos iniciais em Matemática.

A pesquisa realizada por Alencar (2012) oferece uma importante direcionalidade a nosso estudo, uma vez que pesquisadora analisou o conhecimento profissional docente de professores do $5^{\circ}$ ano do ensino fundamental em uma escola que saiu de um índice insatisfatório para no posterior ano conseguir o $5^{\circ}$ melhor resultado em uma avaliação externa no Estado de São Paulo.

Reaplicamos o questionário, entrevista e realizamos nova recolhida de atividades em uma outra escola que obteve o perfil semelhante de superação - ao estudo anterior- nos índices do Saresp Sistema de Avaliação e Rendimento do Estado de São Paulo.

A referida escola apresentou, em 2008, índice de 1,66 e nos anos subseqüentes teve crescente desenvolvimento obtendo no Saresp de 2012 media de 5.58 em uma escala de 0 a 10.

Como sujeito da pesquisa selecionamos um professor do 5o ano do Ensino Fundamental, segmento este avaliado na referida avaliação. O questionário reaplicado é composto por quatro questões do Campo Multiplicativo, que tiveram baixo índice de desempenho desta avaliação externa. Colocamos nas situações protocolos com resoluções de alunos fictícios para que os docentes analisassem e sugerissem intervenções. Especificamente neste estudo apresentaremos um dos exemplos utilizados. Cabe salientar que solicitamos atividades ao docente que foram aplicadas em sala com os seus alunos.

Para explicitar nosso estudo apresentaremos a relevância e os fundamentos teóricos no qual embasamos este artigo e após relataremos sobre os procedimentos metodológicos adotados, apresentaremos a questão do questionário com a análise do professor, aspectos relevantes da entrevista, além das atividades recolhidas e as nossas considerações finais. 


\section{RELEVÂNCIA E FUNDAMENTOS TEÓRICOS}

O interesse em realizar este estudo surgiu com o aparecimento de alguns resultados e encaminhamentos apontados no estudo de Alencar (2012), no qual indica possíveis aspectos e conhecimentos profissionais docentes que contribuíram para um bom desempenho dos alunos em Matemática. Entre estes fatores estão o estudo de materiais de apoio como relatórios, guias de planejamento e outros pelo professor, além de uso de situações contextualizadas no enriquecimento de suas atividades e uso de aluno monitor para os alunos com dificuldade. Com isso procuramos outras instituições bem sucedidas nesta avaliação externa no qual pudéssemos pesquisar quais fatores promoveram tal avanço significativo.

Utilizamos como embasamento teórico os autores que relatam sobre o Conhecimento Profissional Docente: Shulman (1986), Ball e Bass (2003) e Tardif e Raymond (2000).

Observamos nos estudos Shulman (1986) a caracterização do Conhecimento Profissional Docente por três vertentes: o Conhecimento do Conteúdo Específico que trata-se das características e peculiaridades do conteúdo; o Conhecimento Pedagógico do Conteúdo que é o modo de explica-lo para que ocorra o entendimento e compreensão do mesmo e Conhecimento Curricular do Conteúdo que é a relação do conteúdo com os conhecimento anteriores. O autor acrescenta que todos os conhecimentos são complementares e por tanto dependem um do outro para se solidificarem.

Em complemento os estudos de Shulman (1986), os autores Ball e Bass (2003), demonstram que o entendimento do pensamento matemático dos alunos auxilia os docentes na reflexão de sua prática pedagógica. Os autores nos questionam "Qual matemática os professores necessitam saber para ensinar efetivamente?", neste sentido o docente necessita ter domínio do conhecimento especializado do conteúdo.

Tardif e Raymond (2000) relatam que parte do conhecimento e habilidades que os professores possuem sobre os saberes e o modo de ensina-los esta diretamente ligado as suas experiências de vida, quando alunos e educadores. Neste sentido os autores confirmam que estes saberes tem estreita relação com o tempo. Assim, notamos nos estudos desses autores que os mesmos 
desenvolveram um quadro de saberes indicando a relação dos saberes com os lugares de atuação, instrumentos utilizados no trabalho e experiências adquiridas neste período.

Para análise das questões matemáticas nos referenciamos na Teoria dos Campos Conceituais de Vergnaud (1991), que cita que o conceito é formado pela tríade $S$ - situações que dão significado, I invariantes compostas pelos objetos, propriedades e relações e $\mathrm{R}$ que são as representações simbólicas. O autor analisa o desenvolvimento do conhecimento com foco no conteúdo relatando que os mesmos são organizados em campos conceituais, que são operações obtidas com as experiências desenvolvimento da maturidade e aprendizagem que ocorrem em um período de tempo. Existem vários campos conceituais aditivos, multiplicativos, geométricos e outros. Especificamente neste estudo abordaremos o Campo Conceitual Multiplicativo.

\section{PROCEDIMENTOS METODOLÓGICOS}

Escolhemos a escola com base em uma pesquisa nos relatórios de resultados de uma avaliação externa do estado de SP dos anos de 2008 a 2012. Selecionamos uma escola que superou um índice insuficiente em 2008 para um índice satisfatório nos posteriores anos.

Destacamos que a referida escola, no ano de 2008, obteve na disciplina de Matemática o índice de 1,66 e, no ano de 2012, 5,58 em uma escala de 0 a 10. Vemos tal proficiência no referido gráfico: 


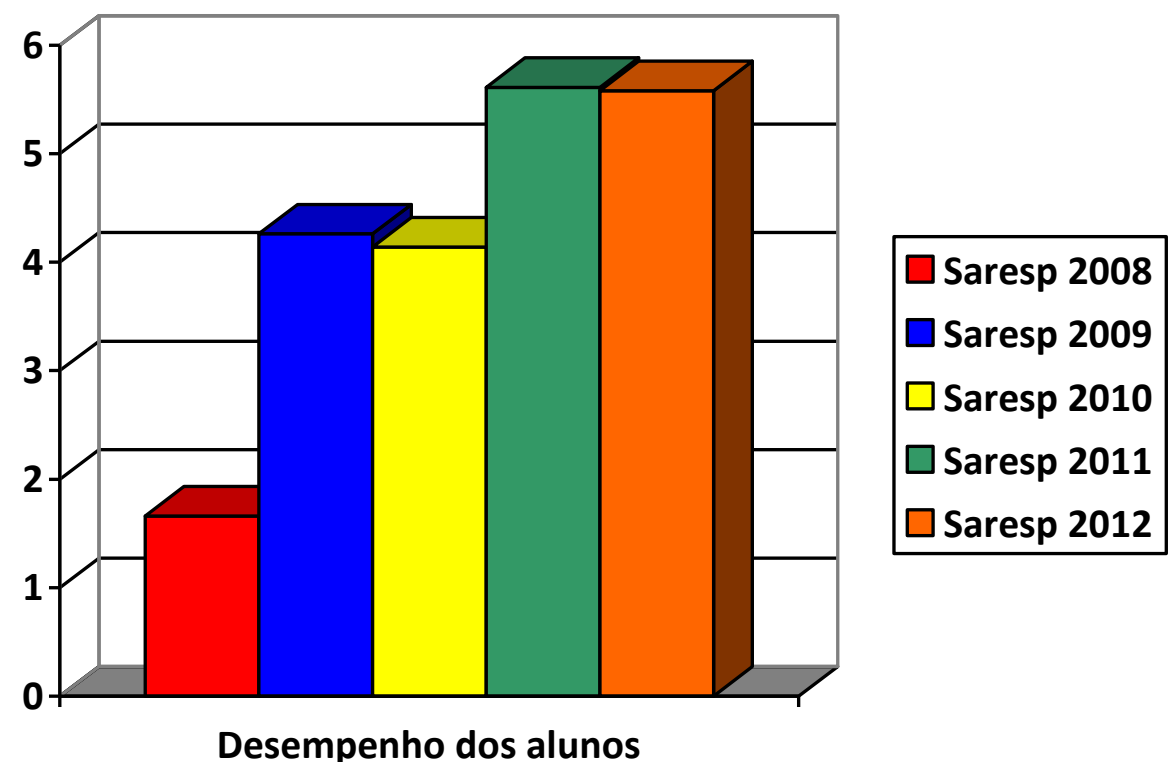

Gráfico 1 - Desempenho dos alunos na Avaliação Externa

Cabe salientar que investigamos quais ações o docente desempenhou para obter tal aprimoramento, como este analisou as produções dos alunos e planejou as suas ações pedagógicas.

Para a coleta de dados reaplicamos um questionário elaborado com questões da referida avaliação externa, no qual os alunos tiveram dificuldade em sua resolução. Cabe destacar que entre estas questões apresentaremos uma abordando a situação problema de proporcionalidade. Para possibilitar uma melhor análise indicamos protocolos com resoluções de alunos fictícios, propondo diferentes soluções que potencialmente poderiam ser realizadas por estudantes. $O$ objetivo foi procurar identificar qual o grau de compreensão do educador acerca de cada resolução nas dificuldades dos discentes e sugerimos ainda que os sujeitos indicassem algumas intervenções pedagógicas. Foi reaplicado também a entrevista, no qual abordou-se alguns aspectos relevantes para a pesquisa e recolheu-se atividades desenvolvidas com os alunos do Professor A. 


\section{DESCRIÇÃO DA QUESTÃO DO QUESTIONÁRIO}

Reiteramos que das quatro questões que compõe o questionário apresentaremos neste artigo uma contendo uma situação de proporcionalidade. Salientamos que esta questão possui como habilidade do Saresp - "Resolver problemas que envolvam a multiplicação e a divisão, especialmente em situações relacionadas à comparação entre razões e a configuração retangular".

Figura 1. Fotos da apresentação dos alunos sobre profissões.

Sabendo-se que 4 maçãs custam RS 2,50. Quanto Julia pagará por 16 maçãs?
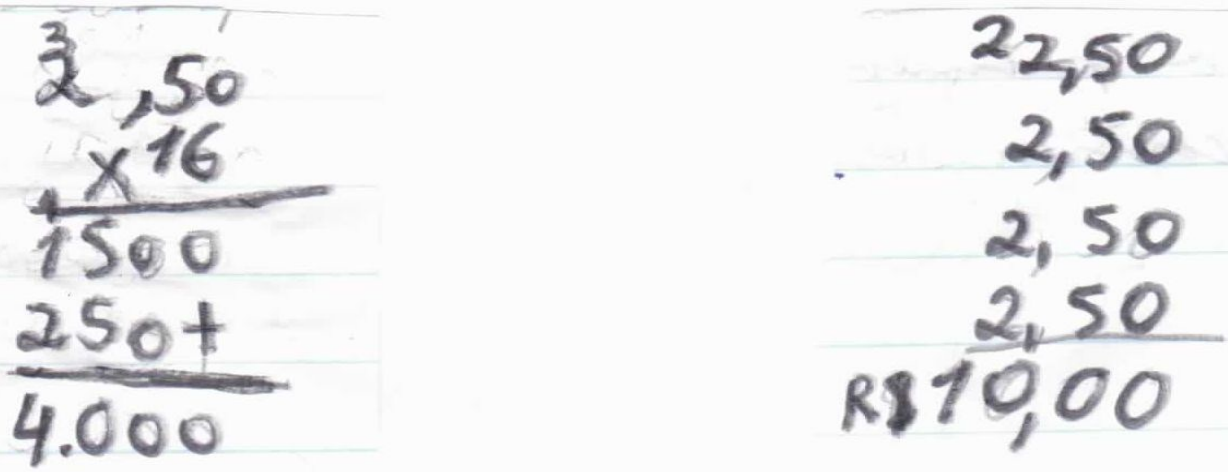

Aluno 1

Aluno 2

A) Explicite aspectos que podem indicar o grau de compreensão de cada um deles sobre a resolução da operação indicada.

B) Dê sugestões para a aprendizagem nos diferentes casos: qual seria sua intervenção?

Fonte: Alencar (2012) 
Para uma melhor análise dos protocolos de resolução escolhidos, explanaremos sobre cada aluno. Analisaremos também a situação problema escolhida.

A questão acima nos indica que não se trata de uma situação-problema convencional, pois verificamos que esta possui um enunciado que propõe a relação proporcional de 4 maçãs com o valor de $\mathrm{R} \$ 2,50$. Cabe destacar que situações como está não são comumente encontradas em livros didáticos e outros materiais pedagógicos que tratam do assunto de modo unitário.

Quanto aos protocolos selecionamos o aluno fictício 1 por cometer um equivoco na interpretação da situação problema. Verificamos que o aluno compreende que o valor de $\mathrm{R} \$ 2,50$ é o de uma maçã, por tanto o mesmo acredita que o valor da proporcionalidade inicia-se na unidade. Inferimos ainda que o aluno se utiliza de experiências anteriores que o fizeram solucionar a questão pelo valor unitário. Aparentemente notamos certo avanço quando o discente utiliza a multiplicação para resolução.

O aluno fictício 2 acerta a questão proposta utilizando o algoritmo da adição. Para Vergnaud (1991), casos como a resolução apresentada do operatório da adição por parcelas iguais indica o que ele considera como Teorema em ação, que é a escolha do estudante da operação para resolução do problema. Percebemos que este aluno entendeu que 4 maçãs custam $R \$ 2,50$ e por isso juntou quatro grupos de quatro para obter as dezesseis. 


\section{ANÁLISE DA QUESTÃO E ENTREVISTA}

Realizamos a pesquisa com um professor do $5^{\circ}$ ano do Ensino Fundamental e obtivemos em sua resposta as seguintes assertivas.

\footnotetext{
“O aluno 1 utilizou a operação mais curta pela multiplicação, mas não percebeu que não era o valor de uma maçã.

O aluno 2 utilizou o processo longo pela adição e acertou a situação." (PROFESSOR A)
}

Observamos que o docente identifica o pensamento do aluno fictício em sua resolução, percebe quais as dificuldades existentes dos mesmos. Este fato nos permite inferir que o docente utiliza práticas aprendidas durante o tempo como citado pelos autores Tardif e Raymond (2000). Vemos este aspecto no relato da entrevista do Professor A:

\footnotetext{
“Estudei no Cefam - Centro de Formação e Aperfeiçoamento do Magistério - e tive uma formação que trabalhou muito o ensino em cima de situações problema, e em percebermos como o aluno pensa." (PROFESSOR A)
}

A assertiva permite identificar como foi a formação inicial deste professor e analisar segundo suas percepções sobre o que os alunos sabem ao realizar a resolução do referido problema, que o docente possui uma boa formação e, por conseguinte uma boa prática pedagógica. Shulman (1986) em seus estudos indica que o conhecimento pedagógico, do conteúdo e curricular estão interligados, com isso um se realiza em detrimento do outro.

Podemos reafirmar tal observação nas intervenções sugeridas pelo docente.

\footnotetext{
“O aluno 1 necessita de atividades de interpretação do problema, analisando o que a situação pede, que operação e utilizando o desenho para interpretar.

$\mathrm{O}$ aluno 2 precisa entender que existem outros modos de resolver o problema, mostraria a multiplicação." (PROFESSOR A)
}

Diante das sugestões notamos o estudo de Ball e Bass (2003) que indaga e descreve qual Matemática o Professor deve ensinar para que a aprendizagem se efetive e como é necessário que 
o docente identifique o pensamento matemático do aluno e utilize dessas observações em sua prática pedagógica, no entanto para tal identificação é preciso o domínio do conhecimento especializado do conteúdo.

Alem disso, percebemos a indicação do que Vergnaud (1991) considera para a formação do conceito quando o professor sugere indicação de outros modos de resolução permitindo que o aluno passe por diferentes experiências de aprendizagem.

Reinteremos que foi realizado uma previa entrevista para identificar o perfil do professor, sua relação com a matemática e analisar quais ações ocorreram para que a escola obtivesse um crescimento do índice de desempenho durante os anos de 2008 a 2012. Neste artigo exporemos as ações de proficiência, citadas a seguir pelo docente:

"Acredito que o bom desempenho dos alunos durante esses anos foi por causa do Ler e Escrever e estudo do material da Secretariada Educação. Agora damos foco em Matemática no Material do EMAI [Projeto Educação Matemática para os anos iniciais do Ensino Fundamental] Junto com essas atividades uso muitas situações problema do dia a dia, algumas tiradas do Saresp antigo, tento trabalhar as situações problemas no oral, demonstrando parte por parte interpretando e peço o registro em desenho do problema." (PROFESSOR A)

Cabe ressaltar que a assertiva nos permite inferir que provavelmente o estudo e utilização do Material fornecido pela Secretária de Educação de modo adequado são fator indicativo do resultado satisfatório nesta escola. Observamos ainda que as complementações de atividades contextualizadas são práticas favoráveis. Este aspecto também foi identificado por Alencar (2012) Quanto a utilização do docente da interpretação dos problemas como estratégia para ensino é observado nos estudos de Borba, Selva, Luna, Silva e Ferreira (2008).

\section{ATIVIDADES DESENVOLVIDAS PELO DOCENTE}

Com intuito de analisar as atividades planejadas pelos professores e desenvolvidas com os alunos em sala de aula, recolhemos atividades do Professor A. 
Figura 1. Atividades do Professor A.

\section{ATIVIDADE 6.1}

Leia as situações abaixo, as quais envolvem vários amigos que gostam de jogar videogame e outras brincadeiras e resolva cada uma delas:

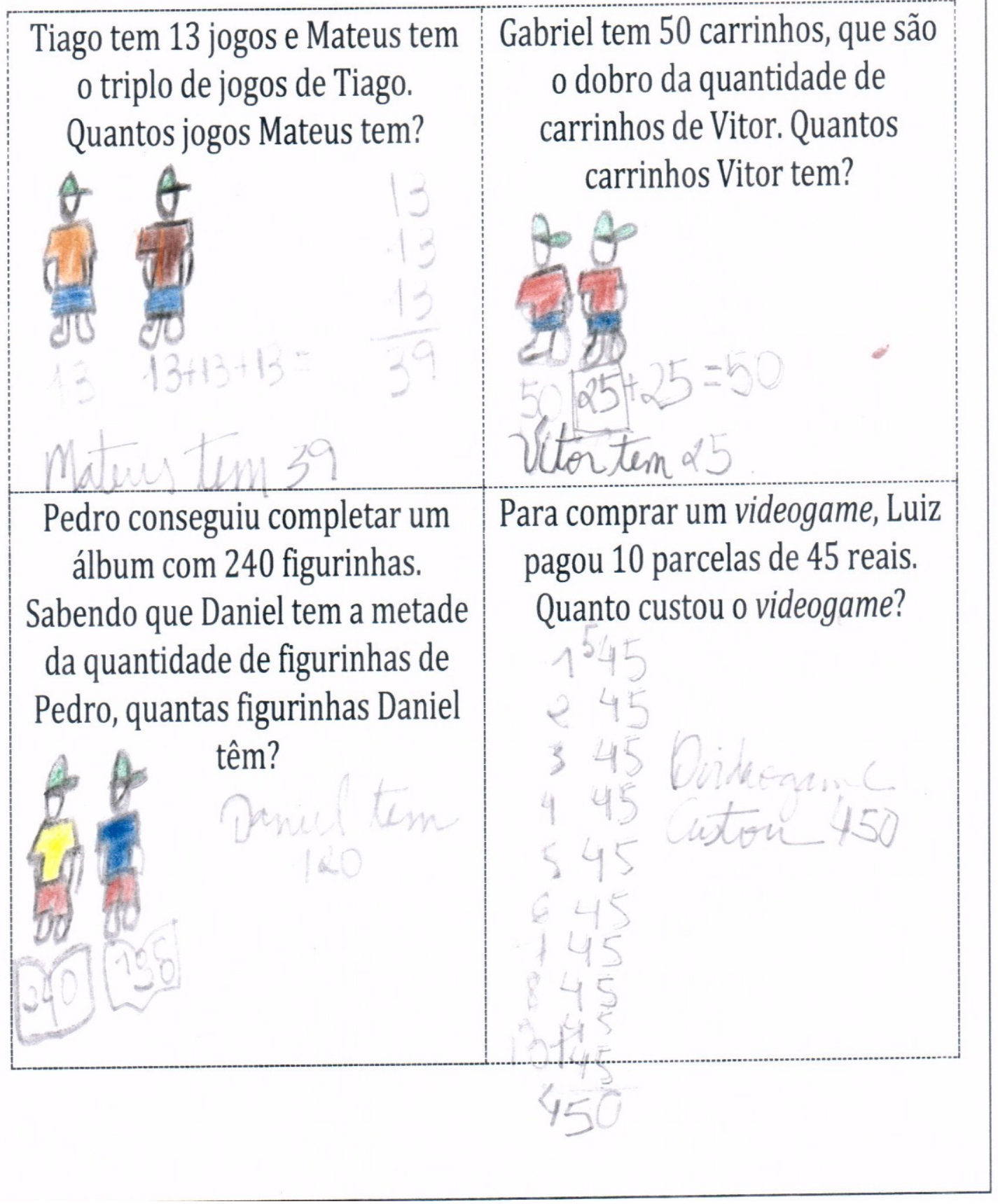




\section{ATIVIDADE 6.2}

Luísa foi a uma loja em que os jogos de videogame estavam em promoção. Eles foram agrupados em kits com 3 jogos diferentes em cada um. Luísa comprou 5 kits. Quantos jogos Luísa comprou?

Luísa viu, próximo ao caixa, uma tabela que mostrava quantidade de kits e os respectivos preços. Ela quis construir uma tabela que apresentasse a quantidade de kits e o número de jogos correspondentes.

Ajude-a a completar os dados que faltam:

\begin{tabular}{|c|c|}
\hline $\begin{array}{c}\text { Quantidade } \\
\text { de kits }\end{array}$ & $\begin{array}{c}\text { Número de } \\
\text { jogos }\end{array}$ \\
\hline 1 & 3 \\
\hline 2 & 6 \\
\hline 3 & 9 \\
\hline 4 & 12 \\
\hline 5 & 1 \\
\hline 6 & 18 \\
\hline 7 & 2 \\
\hline 8 & 24 \\
\hline 9 & 27 \\
\hline 10 & 30 \\
\hline 12 & 3 \\
\hline 15 & 45 \\
\hline
\end{tabular}

- $\quad$ O que você observa na sequência de números que aparecem na segunda coluna da tabela?

O professor nos entregou duas atividades que foram retiradas do $E M A I^{1}$ de $5^{\circ}$ ano, resolvidas por um dos seus alunos. Percebemos que as atividades planejadas estão em consonância com os

\footnotetext{
${ }^{1}$ O Projeto tem como objetivo articular o processo de desenvolvimento curricular, a formação de professores, o processo de aprendizagem dos alunos em Matemática e a avaliação dessas aprendizagens. Retirado do Projeto EMAI $5^{\circ}$ ano.
} 
argumentos apresentados pela docente. As atividades estão próximas ao cotidiano do aluno, pois as situações problemas utilizam da temática brinquedos e brincadeiras. Notamos a utilização de estratégias de raciocínio por meio do desenho, juntamente com o uso do cálculo operatórioadição de parcelas iguais.

Consideramos ainda que atividades como estas possibilitam o desenvolvimento de hipóteses e compreensão do raciocínio matemático, visto que na última questão da atividade 2 é perguntado o que o discente observa na segunda coluna de números da tabela, o mesmo responde que " $A$ primeira coluna é sempre mais um, a segunda coluna e sempre mais três".

\section{CONSIDERAÇÕES FINAIS}

Podemos observar neste estudo que para um bom desempenho dos alunos em Matemática é necessário um conjunto de ações docentes que se complementem para que ocorra a aprendizagem.

Notamos a coincidência de fatores significativos entre o referido estudo e outras pesquisas, como a utilização de modo adequado dos Materiais da Secretaria de Educação e relatórios do Saresp, estudo por parte dos professores destas fontes bibliográficas, complementação com atividades contextualizadas e uso de estratégias de ensino de interpretação de problemas. Estes fatores nos permite inferir possíveis caminhos para a modificação e reflexão da formação continuada dos anos iniciais em Matemática.

\section{REFERÊNCIAS}

ALENCAR, E. S. Conhecimento Profissional Docente de professores do 5o ano de uma escola com bom desempenho em Matemática: o caso das Estruturas Multiplicativas. São Paulo: [Dissertação de Mestrado], 2012.

BALL, D. L.; BASS, H. Toward a practice-based theory of mathematical knowlegde for teaching. In: DAVIS, B.; S. E. E. Procedings of the 2002 Anual Meeting of the Canadian Mathematics Education Study Group. Edmonton: AB:CMESG/GCEDM, 2003. p. 3-14. 
BORBA, R.; SELVA, A.; LUNA, M.; SILVA, D.; FERREIRA, M. Sondando o conhecimento de professoras sobre o desenvolvimento conceitual multiplicativo. 2.o SIPEMAT Simpósio Internacional de Pesquisa em Educação Matemática, Recife, PE, 2008.

SÃO PAULO (ESTADO). Projeto Educação Matemática nos anos iniciais do Ensino Fundamental $5^{\circ}$ ano Secretaria da Educação, Fundação para o Desenvolvimento da Educação, São Paulo: FDE 2012.

TARDIF, M. E.; RAYMOND, D. Saberes, tempo e aprendizagem do trabalho no magistério. Educação \& Sociedade, n. 73, dez. XXI. Revista Educação e Sociedade.

VERGNOUD, La teoría de los campos conceptuales. Recherches en didáctique des mathématiques, p. 133-170, 1991. 